\title{
Yod
}

Revue des études hébraïques et juives

18 | 2013

Le Proche-Orient ancien à la lumière des sciences sociales

\section{La Méditerranée vue par les anthropologues} marxistes

Comment naît une société esclavagiste

The Mediterranean and the Marxist Anthropologists: The Birth of the Slave Society

הים התיכון בעיניהם של אנתרופולוגים מרקסיסטים:לידתה של חברה

\section{Jean-François Bert}

\section{(2) OpenEdition}

\section{Journals}

Édition électronique

URL : https://journals.openedition.org/yod/1749

DOI : $10.4000 /$ yod. 1749

ISSN : 2261-0200

Éditeur

INALCO

\section{Édition imprimée}

Date de publication : 1 septembre 2013

ISBN : 978-2-85831-207-8

ISSN : 0338-9316

\section{Référence électronique}

Jean-François Bert, "La Méditerranée vue par les anthropologues marxistes », Yod [En ligne], 18| 2013, mis en ligne le 08 juillet 2013, consulté le 08 juillet 2021. URL : http://journals.openedition.org/ yod/1749; DOI : https://doi.org/10.4000/yod.1749

Ce document a été généré automatiquement le 8 juillet 2021.

\section{(c) (7) \&}

Yod est mis à disposition selon les termes de la Licence Creative Commons Attribution - Pas d'Utilisation Commerciale 4.0 International. 


\title{
La Méditerranée vue par les anthropologues marxistes
}

\author{
Comment naît une société esclavagiste \\ The Mediterranean and the Marxist Anthropologists: The Birth of the Slave \\ Society
}

הים התיכון בעיניהם של אנתרופולוגים מרקסיסטים:לידתה של חברה

\section{Jean-François Bert}

1 Depuis la publication de La Méditerranée ${ }^{1}$ par Fernand Braudel en 1949, cet "objet» historique, géographique et culturel est devenu central pour les historiens de tout bord. Le modèle braudélien des trois temps a permis, entre autres, à Michel Foucault d'établir sa définition de la notion d'événement dans L'archéologie du savoir, mais surtout de penser la discontinuité. Braudel lui a donné la preuve, historique, qu'il existe, sous les grandes continuités de l'histoire de la pensée, des interruptions, des ruptures et des discontinuités propres aux temporalités historiques.

Ce moment braudélien a cependant presque totalement effacé le fait que ce même espace méditerranéen a été au même moment travaillé par des historiens et des ethnologues attachés, comme Charles Parain (1893-1984) ou André Georges Haudricourt (1911-1996), dans la lignée des travaux de Marc Bloch et de Marcel Mauss sur les techniques, à la matérialité des civilisations, c'est-à-dire aux phénomènes technologiques au sens large comme caractéristique première et décisive de l'humanité et moteur de son histoire.

3 Rationalistes et marxistes, Parain et Haudricourt ont à plusieurs reprises insisté dans leurs travaux respectifs sur l'importance, pour comprendre l'histoire des civilisations, du développement des " forces productives $»^{2}$. Seule l'étude de l'outil, de sa forme, de la manière de le tenir ou de l'utiliser permet, de manière concrète, de caractériser le groupe humain auquel celui-ci appartient. Surtout, Parain et Haudricourt ont cherché à associer de manière parlante et utile - et pas simplement de juxtaposer - des études du 
détail de processus techniques (« comment ça marche ?») à la compréhension d'aspects particuliers des systèmes de pensée et des organisations sociales.

4 C'est tout juste de retour d'un voyage qui l'emmena un an en URSS (1934-1935), d'abord à Leningrad au sein du laboratoire de génétique agronomique du botaniste Nikolaï Vavilov, puis dans certaines stations régionales de cet institut (comme Vladivostok, Krymsk, Volgograd, Pavlovsk), qu'Haudricourt est hospitalisé pour des problèmes pulmonaires au sanatorium de St Hilaire du Touvet en Isère. De son lit, il engage une importante correspondance avec le sociolinguiste Marcel Cohen ${ }^{3}$, mais aussi avec Charles Parain, récent auteur de La Méditerranée. Les hommes et leurs travaux, dans la collection de Géographie humaine, dirigée chez Gallimard par Pierre Deffontaines.

Certains passages de cette correspondance ${ }^{4}$ ont précisément trait à l'Orient méditerranéen :

Que pensez-vous de L'Orient préhistorique de Gordon Childe ?J'y ai trouvé ce que je cherchais sur la préhistoire égyptienne. Entre les civilisations du Fagoum, de Merim $^{5}$ et celle des Badarim il y a un hiatus. La $1^{\circ}$ est produite par des hommes que l'on retrouve actuellement dans les îles et les rives de la Méditerranée. La $2^{\circ}$ est produite par des hommes différents anthropologiquement des précédents, et qui sont les ancêtres des Égyptiens actuels. Ça concorde avec les plantes ${ }^{6}$.

Je n'ai pas encore vu L'Orient préhistorique de Gordon Childe. Mais c'est un spécialiste connu de la préhistoire orientale. Il a fait en particulier au 1er congrès de la préhistoire et de la protohistoire une communication très suggestive sur les 4 écoles de métallurgie qu'il entrevoit, avant -2500 en Égypte, dans l'Égée, en Mésopotamie et dans l'Inde.

Vos considérations sur la brouette et sur les différentes façons de porter les fardeaux m'ont beaucoup intéressé. J'ai quelques notes sur les différentes manières pour les femmes de porter les récipients à l'eau. Mais vous abordez le problème si important des moyens de transport avant la machine avec une ampleur de vues et en même temps une méthode qui à n'en pas douter renouvelleront toute la question ${ }^{7}$.

6 Poursuivant, le 20 novembre 1936, sa tentative de mise au point d'une distinction entre "la civilisation orge - blé dur " (située entre l'Afrique du Nord et la Scandinavie) qui possède la herse et le rouleau, instruments à traction humaine originaires de l'Éthiopie, de l'Égypte et de la Syrie, et la " civilisation du blé tendre » qui, quant à elle, utilise la charrue, inséparable de la traction animale, et dont l'origine se situe dans la vallée de l'Indus, Haudricourt indique :

[...] Remplacés par la charrue dans le domaine méditerranéen, la herse et le rouleau se sont fossilisés dans un emploi spécial (tribulum, pl.) tandis qu'au nord de ce domaine ils ont continué d'avoir un emploi important et ont évolué en se perfectionnant techniquement. Mais il y a l'Égypte - - J'entends encore Vavilov me dire : « il n'y a rien, rien en Égypte » et V. G. Childe explique pourquoi vos Égyptiens historiques sont arrivés très tard en Égypte, je pense qu'ils n'y sont arrivés qu'après l'expansion de la charrue, ils avaient déjà perdu l'usage de la herse-tribulum.

Malgré que les obélisques sont ce qu'on a fait de mieux en fait de menhirs et les pyramides en fait de dolmens, je persiste à croire que l'Égypte ne représente en rien le type de la civilisation orge - blé dur. Les conditions naturelles uniques au monde en font une civilisation non seulement tardive, mais surtout étroitement spécialisée, qui en raison même de son étroite spécialisation, n'a pas eu un rôle important dans l'histoire des techniques.

J'ai entendu dire qu'il y avait des mégalithes en Palestine, mais je n'ai pas retrouvé cela dans le G. Childe; il serait intéressant d'en savoir la date par rapport à l'Égypte. 
Je savais qu'il n'y avait pas de roues en Égypte ancienne, mais n'y a-t-il eu jamais de rouleaux de bois plein. Il me semble difficile d'expliquer sans eux le transport des monolithes.

7 Ce n'est pas un hasard si Parain et surtout Haudricourt se sont tournés vers les ouvrages de Gordon Childe. En effet, l'archéologie leur est indispensable pour mieux cerner la vie matérielle des sociétés et l'archéologie préhistorique est dominée, principalement entre 1925 et 1930, par les travaux de Childe, sous la bannière d'ailleurs d'un marxisme affirmé. Son opposition économique entre d'une part un paléolithique où règnent la chasse et la cueillette et de l'autre un néolithique tourné vers l'agriculture et l'élevage demeure encore aujourd'hui un trait essentiel pour comprendre ces temps passés, même si elle a orienté les recherches archéologiques vers des raisons géographiques, alimentaires et environnementales, plus que culturelles, pour expliquer cette rupture ${ }^{8}$.

Si l'espace méditerranéen est important pour Haudricourt et Parain, c'est qu'il fait au même moment l'objet d'une importante dispute entre les historiens, les orientalistes et les anthropologues russes qui cherchent à actualiser les hypothèses de Marx concernant les sociétés asiatiques pour essayer de comprendre en quoi ce mode de production n'est pas seulement, et comme certains avaient pu le croire, une simple formation de transition entre le mode communiste primitif et le mode antique où l'esclavage règne. Ces discussions féroces, mêlant politique et science, ont connu une réception en France, en particulier dans la communauté des anthropologues français du CERM (Centre d'Étude et de Recherche marxistes) dès la fin des années 1950, à laquelle appartient Charles Parain, mais aussi le géographe africaniste Jean Suret Canale, le sinologue Jean Chesneaux, Pierre Boiteau ou encore Maurice Godelier, sans oublier les historiens Pierre Vidal-Naquet et Jean-Pierre Vernant qui eux aussi ont tenté de montrer le caractère suggestif d'une telle notion élaborée par Marx au détour de trois lettres envoyées à Engels en 1853.

\section{La Méditerranée « antique » de Charles Parain}

9 C'est en 1936 que paraît chez Gallimard, dans la collection du géographe Pierre Deffontaines qui a alors pour but de « reconstituer la vie humaine soit dans un cadre régional déterminé, soit façonnée par un genre de vie spécial », La Méditerranée, les hommes et leurs travaux. Principalement connu pour ses biographies (dont celle de Ramsès II en 1930), Charles Parain va tenter dans ce livre de décrire le domaine naturel de la Méditerranée, tout en montrant la lutte constante des habitants du littoral contre les rigueurs du climat (sécheresse, mais aussi fragilité des forêts, importance de la malaria...).

Le déterminisme géographique n'est qu'une des explications retenues par Parain. Certes, il évoque dans son premier chapitre intitulé «Le domaine méditerranéen » les plissements alpins, le volcanisme, le climat et les vents, les températures et les pluies, l'olivier et le palmier... mais l'historien insiste aussi dans sa qualification de l'espace méditerranéen sur ses ressources végétales, ses innovations techniques (la pêche, la navigation maritime) et l'originalité de ses sociétés rurales (le conflit nomade/ sédentaire, la transhumance, l'assolement biennal imposé par le climat, la monoculture, l'irrigation, le battage des céréales). 
11 Bien peu d'éléments touchent aux rives orientales de la Méditerranée, sauf pour ce qui concerne l'agriculture. Parain est l'auteur, deux ans auparavant, d'un article sur l'agriculture dans l'ancienne Égypte, aussi précise-t-il en 1936 comment le problème de l'irrigation s'est trouvé résolu dans l'Orient ancien de bonne heure, mais que «la solution apportée a eu d'importantes conséquences pour l'organisation politique des États et a fortement contribué à accentuer l'opposition entre les civilisations de l'Ancien Orient et les civilisations méditerranéennes ». Dans le même paragraphe, il poursuit : « Dans le domaine méditerranéen, à l'époque de l'économie paysanne tout au moins, la pratique de l'irrigation ne s'est appliquée qu'à des surfaces peu étendues et ce qui l'a différenciée surtout de ce que l'on trouve dans l'Ancien Orient, c'est qu'au lieu d'être une affaire d'État, elle résulte de l'initiative de communautés restreintes qui en conservent l'administration. ${ }^{9}$

12 Une grande attention est aussi accordée à la question des régimes de propriété, mais surtout, et en bon lecteur de Marx, à certains moyens de production et au mouvement des forces productives. Parain évoque longuement la révolution agricole (chapitre IX) et le développement industriel. Là encore, et comme dans le cas de l'irrigation, ce qui l'intéresse d'abord, ce sont les conséquences sociales et politiques de ces révolutions : «La transformation capitaliste de l'agriculture a brisé les antiques relations. Avec le mélange des populations, chacun devient de plus en plus indifférent à des voisins venus d'ailleurs. Surtout, les différences sociales s'accroissent considérablement [...] les sociétés capitalistes prennent en considération, non pas les êtres humains qu'elles emploient, mais le prix de revient et les bénéfices. $»^{10}$

13 Ce n'est donc pas une surprise si ce livre, malheureusement totalement oublié aujourd'hui ${ }^{11}$, se termine par un chapitre concernant l'évolution du travail industriel, même si cette évolution n'est pas spécifique au monde méditerranéen (passage de l'industrie familiale à l'industrie artisanale et à celle d'atelier), mais qui offre l'occasion à Parain d'évoquer le passé esclavagiste de l'Europe méditerranéenne :

Les pays qui se sont trouvé les premiers à développer les possibilités offertes à l'arboriculture par le sol et le climat, Athènes, les cités grecques les plus évoluées, Carthage, Rome, ont pu accumuler ainsi des richesses qui leur ont permis de constituer de véritables empires et d'acquérir d'immenses troupeaux d'esclaves. L'utilisation de cette main-d'œuvre, provisoirement inépuisable, a accéléré d'une manière étonnante l'évolution des civilisations antiques, a rendu viable une manière de préfiguration du développement intellectuel et politique tel qu'il ne devait se consolider que de nombreux siècles plus tard, à la suite des progrès techniques décisifs réalisés au cours de l'époque féodale. [...] Le système esclavagiste a formé la base matérielle d'une surprenante floraison, avant de précipiter le monde antique dans une ruine définitive. Ce n'est pas le moment d'insister sur ce point. Mais il était nécessaire d'en marquer l'importance : quand on parle des civilisations méditerranéennes, il est essentiel de soigneusement distinguer ce qui, d'une part, constitue pour elles un fond durable et toujours valable - ce qui, d'autre part, n'a été, dans l'histoire des sociétés humaines, qu'une réussite exceptionnelle et transitoire, dont l'éclat ne doit pas masquer la tare congénitale ${ }^{12}$.

14 Si ce passage est important, ce n'est certes pas pour la radicalité du jugement, mais parce que Parain, à l'occasion d'un texte sur le mode de production asiatique écrit au début des années 1960, reprendra cette fois-ci en détail la question de l'esclavage antique qu'il compare à l'esclavage de l'Orient antique. Dans son intervention, l'historien plaide d'abord pour le fait de ne pas confondre, comme l'ont fait jusqu'alors 
certains historiens, le mode esclavagiste de l'Orient ancien avec celui propre à la Méditerranée qui s'est généralisé grâce à l'existence d'une propriété privée du sol, au point de devenir la base "économique» de la communauté antique ${ }^{13}$. Pour Marx, rappelons-le, une des particularités des «sociétés asiatiques» est que l'esclavage se limite au sein des groupes autochtones. Il est également freiné par la possibilité - liée à l'existence d'un État - de disposer en permanence, pour de grands travaux de type hydraulique, du travail des paysans corvéables. Cet esclavage "généralisé " propre à l'Orient antique se limite également, précise Parain, «à l'exécution de gros travaux, le fignolage ou les travaux les plus délicats étant confiés à un petit nombre d'artisans spécialisés dépendants du despote... Tel qu'il se présente avec ses commodités et ses insuffisances, l'esclavage généralisé a rendu possibles d'énormes travaux conduisant à une amélioration parfois considérable des conditions de la production, en premier lieu par la maîtrise de l'eau aussi bien pour l'assèchement que pour l'irrigation $»^{14}$.

Pourquoi Parain décide-t-il de passer par le mode de production asiatique pour expliquer l'esclavage antique ? Plusieurs raisons sont à envisager. Tout d'abord, il s'agit de procéder à la généralisation ${ }^{15}$ de ce mode de production en montrant son usage possible pour décrire d'autres types de sociétés, en l'occurrence les civilisations mégalithique, créto-mycénienne et étrusque qui sont trois civilisations, précise Parain, qui «ont reçu l'impulsion, avec une intensité et une fortune variées, des modèles proposés par les grandes civilisations du Proche-Orient $»^{16}$.

Au niveau théorique, si Parain décide de s'attaquer à la difficile définition du mode de production asiatique, difficile, car extrêmement connotée politiquement, il s'agit pour lui de réfléchir à une autre «notion » marxiste, celle de «communauté primitive ${ }^{17}$, pour essayer de reconstituer, société par société, les divers processus par lesquels l'inégalité s'est introduite dans les sociétés sans classes. En effet, il est important pour Parain, et les autres historiens français du CERM, d'élucider le «mystère » qui entoure la dissolution précipitée de la communauté primitive par la division entre agriculteurs et éleveurs - division soulignée par Childe. En se désagrégeant, cette communauté primitive doit-elle fatalement laisser sa place à la propriété privée ? L'apparition de l'élevage est-elle responsable d'une évolution originale de l'organisation de la production, par rapport aux sociétés sédentaires et agricoles ${ }^{18}$ ?

\section{La place de l'Orient méditerranéen dans les débats sur le mode de production asiatique}

De tous les modes de production définis par Marx, celui asiatique a sans aucun doute possible connu l'histoire la plus mouvementée ${ }^{19}$. Les raisons en sont nombreuses. En premier lieu, la compréhension même du texte de Marx. C'est en 1853, dans trois lettres à Engels, que celui-ci désigne ce mode de production comme possédant une organisation sociale particulière, où l'autorité est représentée par l'assemblée des chefs de famille et par une organisation technique du travail fondée sur la coopération à grande échelle des communautés villageoises dans le but de réaliser de grands travaux d'intérêt général. Marx insiste également sur l'absence de servage et d'esclavage, au sens méditerranéen et antique du terme. La raison de cette différence tient au fait qu'il n'existe pas dans l'Asie antique de propriété terrienne privée. La contrainte politique qui réduit les paysans asiatiques ne peut donc pas être de même nature que l'esclavagisme grec ou romain ${ }^{20}$. 

succession des sociétés tel qu'il est dressé par Marx - il suffit de relire la préface de sa Contribution à la Critique de l'Économie politique (1859) dans laquelle il rappelle que les " modes de production asiatique, antique, féodal et bourgeois moderne apparaissent comme des époques progressives de la formation économique de la société »- ce dernier va susciter pourtant d'importantes réserves. La première vient du fait que certains commentateurs vont chercher à considérer le mode de production asiatique comme un mode de production transitoire qui, historiquement, se situerait entre la communauté primitive, où la population vit de la chasse, de la pêche et, au niveau le plus évolué, de l'élevage et de l'agriculture; et le mode de production antique qui voit l'émergence de la propriété privée et naître l'opposition entre ville et campagne, citoyen et esclave. D'autres vont se demander si la "société " asiatique décrite par Marx en 1853 a réellement existée ${ }^{1}$. D'autres encore vont plutôt chercher à considérer le mode de production asiatique comme une abstraction historique en perpétuelle transformation et qui permet de mettre en relief les traits caractéristiques d'une époque historique.

19 L'orientalise soviétique Vassili Struvé va soutenir par exemple que depuis la plus haute Antiquité, en Égypte, mais aussi en Mésopotamie, les esclaves prisonniers de guerre sont utilisés pour les travaux d'irrigation. Pour lui, la société babylonienne a des caractères proches de celle attique. En 1938, il n'a plus aucun doute sur le fait que ce que Marx désigne par mode de production asiatique correspond en fait à la société de l'Orient antique. Voici l'un des arguments de Struvé que nous tirons du résumé de l'une de ses plus célèbres interventions, intitulée "Définition de la première société de classes » :

Les communautés rurales, signalées dans l'Inde par Marx, ont subsisté longtemps dans l'Égypte ancienne. En Babylonie, elles ont disparu rapidement. Selon Marx, la "population non agricole» des États despotiques de l'Asie ancienne consistait surtout en esclaves et le mode de production correspondait par conséquent à un ordre social mi-esclavagiste, mi-patriarcal. Engels, Lénine et Staline définissaient les premières sociétés de classes comme des sociétés esclavagistes en niant l'existence d'un « mode de production asiatique " particulier ${ }^{22}$.

En remettant en cause l'existence d'une différence entre les États primitifs orientaux et les États plus évolués de la Grèce ou de Rome, Struvé, mais aussi V. Avdiev, en particulier dans son Histoire de l'Orient antique, ouvrent un nouveau champ de recherche pour les historiens russes, du moins jusque dans les années soixante: le panescalavagisme qui permet d'expliquer l'évolution de toute l'Antiquité.

21 A. Tioumenev (1880-1959), au contraire, cherche à combattre toute tentative de transposition de la forme d'esclavage, propre à l'Antiquité gréco-romaine, à l'Orient antique. Il soutiendra l'existence d'une hétérogénéité radicale des deux sociétés et donc de leur esclavage. Pour l'historien, la majeure partie de la production dans l'Orient ancien repose sur la population libre. Il n'existe d'ailleurs qu'une exploitation de la population autochtone et les prisonniers de guerre ne représentent qu'une source secondaire de main-d'œuvre. Tioumenev, dans son Histoire des sociétés esclavagistes antiques (1935), cherche également à distinguer les territoires du Proche de ceux du Moyen-Orient, montrant que les hypothèses de Marx ne valent que pour l'Égypte et la Mésopotamie, deux territoires qui ont des traits communs qui les séparent profondément de l'Antiquité gréco-romaine. Au contraire, la Palestine et la Phénicie 
s'en rapprochent davantage. La différence essentielle entre les États de l'Orient antique et ceux de la Grèce réside principalement dans la structure politique.

C'est entre ces deux voies extrêmes, celle de Struvé et celle de Tioumenev, que les discussions soviétiques concernant le mode de production asiatique et la forme d'esclavage qui en résulte vont se cristalliser jusqu'au milieu des années 1950. Deux tentatives de définition qui ont pourtant en commun d'aborder la définition du mode de production à partir, principalement, de considérations techniques (distribution rationnelle des ressources naturelles), juridiques (absence ou non de propriété foncière) et économiques (commercialisation du surplus).

\section{Haudricourt, l'Asie (tout) contre la Méditerranée} anglo-saxon) consiste à rappeler ce que nous devons aux sociétés orientales, en particulier concernant certaines inventions déterminantes pour le développement de l'Europe: imprimerie, boussole marine, poudre à canon, brouette, horloge hydromécanique, gouvernail d'étambot... De façon extrêmement convaincante, Haudricourt rappelle qu'il n'y a rien dans la langue, l'écriture, la logique ou le mode de pensée chinois qui ne soit un facteur de blocage ou de retard.

Dans un projet d'article intitulé «Recherches des bases d'une étude comparative des mentalités extrême-orientale et occidentale", qu'il rédige en partie lors de son détachement comme bibliothécaire à l'École française d'Extrême-Orient à Hanoï en 1949, l'ethnologue spécialiste des techniques creuse une tentative de comparaison entre Occident et Extrême-Orient qui partirait du « mode d'ascension de l'individu dans la hiérarchie sociale» et "des relations de "classes" sur le plan technologique (organisation pratique du travail) ». Ces deux points de départ lui font apparaître que seule la Méditerranée a connu un stade esclavagiste. Un stade, ajoute-t-il en faisant référence aux débats concernant le mode de production asiatique, qui a été " escamoté » en Chine et qui n'a pas eu lieu en Inde. Haudricourt explique cette présence de l'esclavagisme en Europe d'abord par le fait que l'espace méditerranéen est double : Égypte et Mésopotamie d'une part, Iran de l'autre. La conséquence de cette diversité a été l'usage du commerce, des relations, des activités de tous ordres intensifiées (alphabet - civilisation égéenne - etc.) : « Le morcellement de la Grèce et de la Méditerranée, facilitant des contacts nombreux et l'expansion de la civilisation, a par là même, rendu possible l'épanouissement de la société esclavagiste: les "voisins" étaient de même civilisation (même niveau technique) donc utilisables comme esclaves $»^{24}$.

$\mathrm{Au}$ contraire, si la Chine n'a pas connu cette configuration, c'est en grande partie dû au fait que sa géographie n'a pas permis l'épanouissement de la mentalité commerciale et de l'esclavage. 
Une autre différence essentielle importe aussi à Haudricourt. Il s'agit de comprendre le rôle joué par l'élevage du bétail dans le développement d'une mentalité européenne. En effet, alors que l'on n'hésite pas à frapper une vache en Europe, en Indochine et dans le Proche-Orient, le rapport homme-animal est inversé :

Un petit gosse mène paitre le buffle, et c'est le buffle qui le défendra du tigre, tandis qu'en Occident, c'est le berger qui protège les moutons du loup. En Indochine, les cochons se gardent tous seuls, le fils prodigue serait au chômage. En Occident, le berger commande, protège son troupeau; origine de la mentalité paternaliste; le berger sait mieux que le mouton ce qu'il faut à celui-ci comme pâture; c'est ce qui a permis le développement de l'esclavage dans l'antiquité : bien sûr, en Chine, le mandarin est "père et mère " du peuple, mais il ne commande pas, comme les parents ne commandent pas à leurs enfants qui sont insupportables ${ }^{25}$.

Cette différence se retrouve pour Haudricourt dans le traitement horticole. En Orient on ne touche pas à la récolte, «il n'y a pas à frapper sur elles ni à taper sur elles». En Occident, c'est techniquement que l'on tente de diriger la nature. Une pratique qui pour Haudricorut n'a eu que pour conséquence de durcir les rapports humains, développant une mentalité de pasteur.

La conclusion à laquelle arrive Haudricourt en 1949 n'est pas sans rappeler celle que Parain avait esquissée en 1936 :

L'économie pastorale ne pouvait s'installer qu'à l'ouest (climat sec) et ne pouvait avoir son maximum d'influence religieuse (Palestine) et économique (PhénicieEgée) que là. En Chine, l'éleveur est resté en marge, les Turco-Mongols ne sont sortis que récemment de la forêt, ils n'ont pu fournir que quelques dynasties vite absorbées. Les Indo-Européens étaient les vrais pasteurs d'Asie, mais du côté Extrême-Orient, ils ne se sont pas heurtés aux vieilles villes et agriculture d'Asie. Ils n'ont pas extériorisé en une «bible» leur mentalité; à l'ouest, l'élevage s'est répandu et fondu dans l'agriculture européenne... Dans l'Inde, civilisation urbaine antique sur l'Indus, en contact avec la forêt tropicale du Gange : quand il y a contact entre deux civilisations très différentes, le réflexe «normal» est le racisme (Européens aux colonies) donc castes (voir ci-dessus pour expliquer la transmigration, ce que nous disons de la mentalité canaque, M. Leenhardt, Do Kamo.... ${ }^{26}$.

L'ethnologue reprendra à deux autres occasions son esquisse de comparaison. La première fois, en 1954, utilisant un pseudonyme dans la revue France-Asie ${ }^{27}$. La seconde fois, dans la revue de Claude Lévi-Strauss, L'Homme, où il décidera de creuser la différence écologique qui sépare les deux civilisations : un Occident qui serait placé sous le signe de l'éleveur et un Orient sous celui de l'agriculteur ${ }^{28}$. Comme en 1949 , l'opposition que dessine Haudricourt n'est pas seulement technique. Elle se projette dans les aptitudes, les conduites, les tendances et finalement la « vision du monde » de ces civilisations. Morale, religion, droit et philosophie sont directement déterminés par cette différence. Il n'est donc pas absurde, conclut Haudricourt, « de se demander si les dieux qui commandent, les morales qui ordonnent, les philosophies qui transcendent n'auraient pas quelque chose à voir avec le mouton, par l'intermédiaire d'une prédilection pour les modes de production esclavagiste et capitaliste, et si les morales qui expliquent et les philosophies de l'immanence n'auraient pas quelque chose à voir avec l'igname, le taro et le riz, par l'intermédiaire des modes de production de l'Antiquité asiatique et du féodalisme bureaucratique $»^{29}$. 


\section{Conclusion}

La réflexion que Parain a engagée sur la Méditerranée au milieu des années trente, comme celle qu'Haudricourt réalisera à partir de 1949, en tentant de comparer les mentalités occidentales et celles extrême-orientales, ont résolument ouvert la voie à de nouvelles hypothèses théoriques concernant la complexité et la diversité du développement historique des sociétés traditionnelles et ce, à partir d'une prise en compte nouvelle des conditions écologiques et technologiques, comme les processus de domestication animale et végétale.

Le $1^{\mathrm{er}}$ janvier 1937, Haudricourt débute ainsi l'une de ses lettres à Charles Parain :

Je comprends fort bien que pour des raisons pratiques, matérielles, vous ayez été obligé de limiter vos recherches à la Région méditerranéenne, mais je pense que cela a des inconvénients théoriques, et c'est même le principal reproche que je ferais à votre livre sur la Méditerranée. Il y aurait moins d'inconvénients pour les régions centrales de l'humanité comme l'Inde ou la Chine, ou pour les régions isolées comme l'Amérique ou l'Australie; mais l'Europe est un finistère, un Extrême-Orient, qui doit précisément sa chance d'avoir été la moins isolée des régions périphériques de l'humanité.

Pouvait-il imaginer que moins de trente ans plus tard, les historiens de l'Antiquité méditerranéenne utiliseront pleinement les considérations de Marx sur la société asiatique pour expliquer la naissance et l'expansion de l'esclavage en Méditerranée ?

\section{NOTES}

1. Le sujet de Braudel a été déposé en 1923 sous le titre Philippe II, l'Espagne et la Méditerranée au $\mathrm{XVI}^{e}$ siècle. Si l'on en croit la préface à la seconde édition, la problématique aurait été établie dans ses grandes lignes avant la guerre: «La Méditerranée ne date pas de 1949, année de sa publication, ni même de 1947, année où elle fut soutenue comme thèse, en Sorbonne. Elle était fixée dans ses grandes lignes, sinon écrite entièrement, dès 1939, au terme de la première jeunesse éblouissante des Annales de Marc Bloch et de Lucien Febvre, dont elle est le fruit direct». Fernand Braudel, "Préface à la seconde édition", La Méditerranée et le monde méditerranéen à l'époque de Philippe II. 1. La part du milieu, Paris, Armand Colin, 1990. Selon Paule Braudel, Lucien Febvre, directeur de cette thèse, reçoit une première version en 1941, une seconde en 1942. Voir Paule Braudel, "Les origines intellectuelles de Fernand Braudel: un témoignage ", Annales. Économies, Sociétés, Civilisations, vol. 47, n 1, 1992, p. 237-244. (http:// www.persee.fr/web/revues/home/prescript/article/ahess_0395-2649_1992_num_47_1_279038)

2. Celles-ci se composent, selon Karl Marx, des ressources de la nature, des moyens de travail et du procès de travail.

3. Jean-François Bert, «A. G. Haudricourt et M. Cohen : un regard singulier sur la linguistique », Langages et sociétés $\mathrm{n}^{\circ} 128,2009 / 2, \mathrm{p} .77-98$.

4. Cette correspondance a fait l'objet d'une transcription partielle dans le volume Penser le concret, A. G Haudricourt, A. Leroi-Gourhan et C. Parain, dir. Noël Barbe et Jean-François Bert, Paris, CREAPHIS, 2011, p. 147-163. 
5. Il s'agit des cultures archéologiques prédynastiques Fayoum A (5200-4000 av. n. è.), Merimde (4800-4300 av. n. è.) et Badari (4400-4000 av. n. è.). Haudricourt orthographie mal les noms. Parmi ses sources à ce sujet figure le livre de Guy Brunton, Gertrude Caton-Thompson, The Badarian Civilisation and Predynastic Remains near Badari, vol. 46, Londres, British school of archaeology in Egypt and Egyptian research account, 1928.

6. Lettre de Haudricourt à Parain, 22 octobre 1936.

7. Réponse de Parain à Haudricourt, 6 novembre 1936.

8. Voir Gordon Childe, L'Orient préhistorique, Paris, Payot, 1935. C'est dans ce livre que Childe aborde les principaux mécanismes de diffusion et dessine les deux grandes révolutions connues par l'humanité : en premier, le fait que l'homme passe du stade où il cherche sa nourriture au stade où il la produit. En second, la naissance de l'économie urbaine qui fait naître l'industrie et le commerce.

9. Charles Parain, La Méditerranée. Les hommes et leurs travaux, Paris, Gallimard, 1936, p. 118-119.

10. Ibid., p. 172.

11. Voir la très belle analyse de Noël Barbe, «Dans un papier kraft... Braudel lu par Charles Parain ou Marx à la trace » dans Penser le concret, op. cit. p. 33-57.

12. Ibid., p. 209.

13. Voir les travaux de Yvon Garlan et en particulier Les esclaves en Grèce ancienne, Paris, La Découverte, 1984. Un livre par ailleurs dédié à Charles Parain.

14. Charles Parain, «Protohistoire méditerranéenne et mode de production asiatique » dans Sur "le mode de production asiatique », Paris, CERM, Éditions sociales, 1969, p. 169-195, p. 172.

15. Les tentatives de ce type sont nombreuses. En 1958, Jean Suret-Canale tente d'exporter la définition du mode de production asiatique dans le but de décrire le fonctionnement de certains États d'Afrique occidentale précoloniale. Pierre Bonte, quant à lui, cherche à dépasser l'interprétation de Marx et d'Engels grâce à de nouvelles données venant de description des Touaregs Kel Gress du Niger - données qui concernent l'organisation domestique. Jean Chesneaux tente lui aussi un rapprochement entre le mode de production asiatique et les anciennes sociétés précolombiennes. À ces quelques exemples, il faut ajouter les tentatives de Pierre Boiteau pour la société malgache précoloniale, ou encore celle d'Hélène AntoniadisBibicou pour la Byzance de la période préféodale.

16. Charles Parain, « Protohistoire méditerranéenne et mode de production asiatique », p. 170.

17. C'est à la fin des années 1950 , suite à la visite de l'orientaliste sinologue Ferenc Tokeï venu présenter les traits généraux de la forme de propriété asiatique, que les discussions concernant le mode de production asiatique débutent en France. En 1964, la revue La Pensée publie un premier dossier rassemblant un chapitre de l'exposé de Tokeï, une présentation de Charles Parain, des «remarques » de Jean Chesneaux et une bibliographie commentée par M. Godelier. L'ensemble du manuscrit de Tokeï, intitulé «Sur le mode de production asiatique », est publié en 1966. Un an plus tard, la revue Recherches internationales ( $n^{\circ}$ 57-58) publie un volume sur les "Premières sociétés de classes et mode de production asiatique » qui rassemble une large part des textes russes concernant l'évolution des sociétés précapitalistes. En 1969, le CERM donne à lire aux Éditions sociales le résultat de ses séminaires dans Sur le mode de production asiatique. Ce collectif va connaître plusieurs rééditions successives, jusqu'au milieu des années soixante-dix. A cela, il faut ajouter un article de Maurice Godelier dans Les Temps modernes, intitulé «Le mode de production asiatique ", mai $1965, \mathrm{n}^{\circ} 228$, p. 2002-2027; un autre numéro de la revue La Pensée contenant des textes de Pierre Boiteau et de Suret-Canale, La Pensée $n^{\circ} 117$, octobre 1964 ; et enfin le texte de Vidal-Naquet sur Wittfogel qui paraît dans la revue des Annales, "Histoire et idéologie : Karl Wittfogel et le concept de "mode de production asiatique" ", Annales Économies, Sociétés, Civilisations, vol. 19, n 3, 1964, p. 531-549. (http://www.persee.fr/web/revues/home/ prescript/article/ahess_0395-2649_1964_num_19_3_421177) 
18. Pour les anthropologues du CERM, dont Parain et plus encore Haudricourt, il s'agit d'évaluer la place de la domestication dans la marche d'affranchissement de l'homme des différentes contraintes naturelles.

19. Une autre controverse tout aussi importante concerna au début des années 1950 la question du passage de la féodalité au capitalisme. Controverse qui débute avec le livre de Maurice Dobb, Studies in The Development of Capitalism, Londres, Routledge - Paul Kegan, 1946, dans lequel l'auteur cherche à comprendre à quel moment et par quels moyens on peut dire que le mode de production féodal a disparu. Controverse importante qui impliqua d'abord la définition du concept de mode de production. Voir Maurice Dobb et Paul M. Sweezy, Du féodalisme au capitalisme : problèmes de la transition, Paris, Maspero, 1977.

20. Marx-Engels Gesamtausgabe, Dritte Abteilung - Des Briefwechsel zwischen Marx und Engels 1844-1853, Verlag Detlev Auvermann KG, 1970. (Lettre du 2 juin 1853 : p. 474-478; Lettre du 6 juin 1853 : p. 478-482; Lettre du 14 juin 1853 : p. 483-487). Voir Marx/Engels, Trois lettres à propos du mode de production asiatique, Strasbourg, Les carnets, 2009.

21. C'est en 1931 que le mode de production asiatique est officiellement considéré comme une variante asiatique du féodalisme d'Europe occidentale.

22. Pour en savoir plus sur cette dispute, voir Jan Percika, « Discussions soviétiques ", Recherches internationales $\mathrm{n}^{\circ}$ 57-58, 1967, p. 59-78.

23. Dans son Science and Civilization in China (dont le premier numéro "Introduction. Orientations» est publié en 1954), Joseph Needham va ouvrir également de nouvelles perspectives comparatistes en étudiant et en répertoriant la pensée, la science et les techniques chinoises sur plus de vingt siècles. Une pensée qui a pour caractéristique principale d'être holistique et organisatrice de l'univers, algébrique et non géométrique (à la différence de la science grecque), et fondée sur l'observation et l'expérimentation.

24. A.G. Haudricourt, « Recherches des bases d'une étude comparative des mentalités extrêmeorientale et occidentale ", écrit en 1949 et édité dans Des gestes aux techniques, Paris, MSH, 2010, p. 167-177.

25. Ibid., p. 175.

26. Ibid., p. 176.

27. L'article intitulé «Essai sur les origines des différences de mentalités entre Occident et Extrême-Orient " a été publié pour la première fois dans la revue France-Asie. Revue mensuelle de culture et de synthèse franco-asiatique en juillet 1954, $n^{\circ} 98$, p. 814-824. Haudricourt signa cet article du pseudonyme René de Hétrelon. (Repris dans Des gestes aux techniques, op. cit., p. 167-177.)

28. Voir A.G. Haudricourt, «Domestication des animaux, culture des plantes et traitement d'autrui », L'Homme, vol. 2, n 1, 1962, p. 40-50 (repris dans Des gestes aux techniques).

29. Ibid., p. 50.

\section{RÉSUMÉS}

La parution de la Méditerranée de Braudel a presque totalement effacé le fait que ce même espace a été au même moment travaillé par des historiens et des ethnologues attachés, comme Charles Parain (1893-1984) ou André Georges Haudricourt (1911-1996), dans la lignée des travaux de Marc Bloch et de Marcel Mauss sur les techniques, à la matérialité des civilisations, c'est-à-dire aux 
phénomènes technologiques au sens large comme caractéristique première et décisive de l'humanité et moteur de son histoire.

Contemporary to the monumental Braudel's work on the Mediterranean, few other historians and ethnologists were working on a similar topic form a Marxist perspective. In my article I analyze the major contribution of Charles Parrain (1893-1984) and André Georges Haudricourt (1911-1996) to the explanation of the oriental mode and the slave society of production by emphasizing the role of the technology and the materiality.

INDEX

Mots-clés : mode de production oriental, société esclavagiste, pensée marxiste, Parain Charles (1893-1984), Haudricourt André Georges (1911-1996)

Index géographique : Méditerranée, Proche-Orient

\section{מילות מפתח}

הליכי ייצור מזרחיים, המזרח התיכון, הים התיכון, חברה משעבדת, מחשבה:

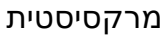

Keywords : oriental mode of production, slave society, Marxist theory, Parain Charles (1893-1984), Haudricourt André Georges (1911-1996), Mediterranean, Middle East

\section{AUTEUR \\ JEAN-FRANÇOIS BERT}

Université de lausanne 